\title{
Guest Editors' Editorial: Mesostructured Materials: Design and Opportunities
}

\author{
Bao-Lian Su · Joël Patarin
}

Published online: 8 October 2009

(C) Springer Science+Business Media, LLC 2009

Since the 1990s, mesoporous solids and mesostructured materials have been extensively studied, and this field has been increasingly broadened to the extent that it has received significant attention from the scientific community. ScienceWatch.com has recently examined the literature on mesoporous materials over the past decade and over the past 2 years using only the specific term "mesoporous". In June 2008, the resulting database contained 6,813 (10 years) and 2,422 (2 years) papers; 11,168 authors; 74 nations; 504 journals and 1,970 institutions. This analysis indicated that over the last 10 years, the concentration appears to have been on synthesis, structures and catalytic applications of mesoporous materials. Over the last 2 years, the focus of the most-cited papers turns to the numerous applications of mesoporous materials. These applications include opto-electronic devices, drug-delivery systems, bone-tissue engineering and other potential uses in the medical, clinic toxicology and environmental fields. However, a void in real industrial applications still exists.

\section{B.-L. Su ( $\square)$}

Laboratory of Inorganic Materials Chemistry (CMI),

The University of Namur (FUNDP), 61 rue de Bruxelles,

5000 Namur, Belgium

e-mail: bao-lian.su@fundp.ac.be

\section{B.-L. Su}

State Key Laboratory of Advanced Technology for Materials Synthesis and Processing, Wuhan University of Technology, 122 Luoshi Road, Wuhan, Hubei 430070, China

\section{J. Patarin}

Equipe Matériaux à Porosité Contrôlée (MPC), Institut de Science des Matériaux de Mulhouse (IS2M), LRC CNRS 7228, Université de Haute Alsace (UHA), ENSCMu, 3 rue Alfred Werner, 68093 Mulhouse Cedex, France e-mail: joel.patarin@uha.fr
The 1992 discovery of mesoporous materials by researchers at Mobil Oil Corporation was followed by several meetings devoted entirely to the science of these exciting solids. The first five symposia were held in 1998 in Baltimore (USA), in 2000 in Québec (Canada), in 2002 in Jeju (Korea), in 2004 in Cape Town (South Africa) and in 2006 in Shanghai (China). The last symposium was held in Namur in September 2008 and was jointly organized by the Laboratory of Inorganic Materials Chemistry at the University of Namur, Belgium and the Laboratory of Controlled Porosity Materials at the University of Haute-Alsace, France under the auspices of the International Mesostructured Materials Association (IMMA). In this symposium, six main sessions dealing with oxide mesostructured materials, non-oxide mesostructured materials, hierarchical mesostructured materials, biological and medical applications, mixed topics, bioinspired and other new synthesis routes to mesostructured materials and applications in catalysis, separation processes, etc. have been addressed.

The aim of IMMS2008 meeting was to review the progress of the latest results in the field of mesostructured materials. The focus was made on interdisciplinary research approaches (complexity and relations with other disciplines, in particular, biological, pharmaceutical, environmental and medical fields), potential for the future and the promotion of the field in industry (design and promise), promotion of young scientists (new leaders in the field) and on future directions (new trends). This meeting provided an active discussion forum to debate the needs of our modern society and human life. The IMMS2008 began with a lecture given by Nobel Laureate Prof. Jean-Marie Lehn and then offered 7 plenary lectures, 6 keynote lectures, 5 invited lectures and 38 oral presentations and 375 posters (including recent research reports). This meeting attracted over 400 participants from more than 40 countries. At this occasion we would like to thank all the sponsors (University of Namur-FUNDP, 
Université de Haute Alsace, FNRS (Belgium), CNRS (France), 3L International, Dataphysics, DGA, IFP, Lhoist, Micromeritics, Night and Day Press, Olmix, Panalytical, Perkin-Elmer, Quantachrome, Sopra, Royal Society of Chemistry-Namur division).

This special issue presents a series of selected papers written by leading experts in the field who participated in IMMS2008. The special issue is organized in two mains sections. One focuses on the synthesis of oxide and nonoxide mesostructured materials with an aspect relative to the shaping of these solids and the development of new synthesis routes. The second section relates to their applications for metal removal, in electrochemistry, biomimetic and red/ox catalytic applications.

The Editors of this special issue would like to acknowledge the authors of all contributions without which the Special Issue would have never been possible. We also are grateful to the referees for their in-depth comments made to the submitted manuscripts. Our gratitude goes to Springer for the opportunity to highlight the work in this important scientific field and for their cooperation in preparing this special issue. 